Ciencia y Educación, Vol. 5, No. 3, septiembre-diciembre, 2021

ISSN (impreso): 2613-8794・ISSN (en línea): 2613-8808

DOI: https://doi.org/10.22206/cyed.2021.v5i3.pp19-38

\title{
Educabilidad y salud mental de universitarios salvadoreños durante la pandemia por Covid-19
}

\author{
Educability and mental health status of Salvadoran university \\ students during the Covid-19 pandemic
}

Fernando José Mena a ORCID: 0000-0003-2474-3194

Verónica De Paz ${ }^{\mathrm{b}}$ ORCID: 0000-0002-7886-7478

Mario Avilés ${ }^{c}$ ORCID: 0000-0001-5985-7226

Lucía Orantes ${ }^{\mathrm{d}}$ ORCID: 0000-0003-1096-1019

Recibido: 6/04/2021 Aprobado: 7/06/2021

Cómo citar: Mena, F. J., De Paz, V., Avilés, M., \& Orantes, L. (2021). Educabilidad y salud mental de universitarios salvadoreños durante la pandemia por Covid-19. Ciencia y Educación, 5(3), 19-38. Doi: https://doi.org/10.22206/cyed.2021.v5i3.pp19-38

\section{Resumen}

Esta investigación tiene como objetivo establecer las condiciones de educabilidad (es decir, acceso tecnológico, determinantes personales y determinantes sociales) y la presencia de sintomatología de depresión, ansiedad, estrés e insomnio en los universitarios salvadoreños durante la pandemia por Covid-19. El estudio cuantitativo transversal de alcance correlacional contó con una muestra de 1,550 universitarios salvadoreños de 25 instituciones, quienes respondieron un cuestionario en línea. La mayoría percibió condiciones poco favorables de educabilidad, excepto en la subescala de acceso tecnológico; el $88.5 \%$ está en riesgo clínicamente significativo de experimentar al menos uno de los estados emocionales estudiados y el 45.4\% de los cuatro estados combinados. También, los modelos predictivos evidencian que mejores condiciones

\begin{abstract}
This study aims to establish the educability conditions (i.e., technological access, personal determinants, and social determinants) and depression, anxiety, stress, and insomnia symptoms among Salvadoran university students during the Covid-19 pandemic. This cross-sectional quantitative study of correlational scope had a sample of 1,550 Salvadoran university students from 25 institutions, who answered an online survey. Most of them perceived adverse educability conditions, except in the technological access subscale; $88.5 \%$ would be at a clinically significant risk of experiencing at least one of the emotional conditions inquired, and $45.4 \%$ of the four of them combined. Also, the predictive models indicate that better educability conditions predict fewer emotional symptoms. In addition, the most vulnerable groups to these adverse effects are women, students
\end{abstract}

\footnotetext{
a Investigador asociado de la Fundación Pro Educación de El Salvador (FUNPRES), El Salvador.

Correo-e: fernandomena@funpres.org.sv.

b Psicóloga de la Fundación Continúa, El Salvador. Correo-e: 00222814@uca.edu.sv.

c Profesional independiente, El Salvador. Correo-e: 00111215@uca.edu.sv.

d Estudiante de la licenciatura en Psicología, Universidad Centroamericana José Simeón Cañas, El Salvador.

Correo-e: 00119617@uca.edu.sv.
} 
de educabilidad pronostican menores síntomas emocionales. Además, los grupos más vulnerables a estos efectos adversos son las mujeres, los estudiantes de la universidad nacional, los jóvenes y quienes reportan casos de contagio por Covid-19 en su familia.

Palabras clave: aprendizaje en línea, enseñanza superior, salud mental, pandemia, grupos desfavorecidos.

\section{Introducción}

En el contexto de la enfermedad del coronavirus 2019 (Covid-19), el 11 de marzo de 2020 el Gobierno de El Salvador suspendió clases en todas las instituciones educativas, incluyendo las Instituciones de Educación Superior (IES) (Deutsche Welle [DW], 2020a), que aglutinan a más de 200,000 estudiantes en 41 instituciones (Instituto de Acceso a la Información Pública [IAIP], 2020). Ante esta situación, las IES se vieron en la necesidad de adaptar los planes de estudio y de formación a módulos o asignaturas compatibles con la virtualización de los procesos educativos, lo que requirió de una alta inversión en recursos tecnológicos y formación docente y estudiantil (Tobar, 2020). Por lo menos hasta mayo de 2021, las clases presenciales todavía se encuentran suspendidas, luego de aplazar el regreso paulatino a actividades presenciales en enero de 2021 por un alza en los casos de Covid-19 en el país (Presidencia de El Salvador, 2021).

\section{Enseńanza Remota de Emergencia y Educabilidad}

La transición a metodologías de enseñanza a distancia de manera abrupta e instantánea se denomina Enseñanza Remota de Emergencia (ERE), que en el contexto de la pandemia se ha llamado coronateaching. Este cambio incluyó una mudanza rápida del aula de clases a plataformas de videollamadas, sustituyó el material impreso por $p d f$ 's, archivos multimedia, podcasts y otros recursos virtuales; y obligó a estudiantes a comunicarse con sus pares y profesores mediante correos electrónicos y videollamadas. Pero dada su ejecución de emergencia, el coronateaching busca implementar clases at the national university, young students and those who report Covid-19 contagion cases in their family.

Keywords: Online learning, post-secondary education, mental health, pandemic, vulnerable groups.

virtuales sin cambiar el currículum ni la metodología utilizada en modalidad presencial; es decir, se cambia el canal, pero no la forma de enseñar (Pérez, 2020).

Es posible que la ERE haya impactado fuertemente en las condiciones de educabilidad de las y los estudiantes, es decir, en su capacidad para apropiarse del conocimiento y acceder a los recursos y las aptitudes que les permiten ser parte del proceso educativo (Bonal \& Tarabini, 2016; Rodelo \& Castro, 2016). Esto, junto a las condiciones económicas adversas y la propia amenaza del virus, puede representar una amenaza a la carrera universitaria de las y los estudiantes, la que perciben como un factor clave para su independencia económica, logro de metas personales, emancipación de su núcleo familiar y crecimiento financiero (Villa, 2016).

Mediavilla y Gallego (2016) mencionan que la educabilidad es un indicador explicativo o causal del rendimiento académico. Este se refiere a las calificaciones o los logros, resultados del aprendizaje adquirido, y como un proceso activo que también involucra la proactividad del estudiantado (Tipismana, 2019). Barahona (2014) clasifica los determinantes del rendimiento académico en dos aspectos: (a) personales, refiriéndose a características propias del individuo, como la inteligencia, las aptitudes, la motivación y la asistencia a clase; y (b) sociales, es decir a las condiciones externas a la persona que afectan su rendimiento, como el contexto socioeconómico y familiar y las variables demográficas. Estos, junto al acceso a los recursos tecnológicos (propios de la ERE), son procesos que pueden estar negativamente afectados en el contexto de la pandemia. 
Condiciones de educabilidad afectadas por la pandemia de Covid-19

Las y los estudiantes reportan que el acceso a la tecnología necesaria ha sido un factor decisivo para continuar con su ańo académico, debido a su capacidad económica y a los recursos en casa (Peña, 2020). Algunos estudiantes reconocen deficiencias en el manejo de herramientas web, señalan que han tenido que hacer mejoras en su servicio de internet y comentan que no cuentan con dispositivos adecuados para recibir clases ni el software esencial para aprender (Cortéz \& Campos, 2020; Torres et al., 2020). En este contexto, se reporta un aumento de la brecha digital entre hombres y mujeres, tanto en el acceso a los dispositivos necesarios como en su conexión a internet, que perjudica más a estas últimas (Comisión Interamericana de Mujeres y Organización de Estados Americanos [CIM-OEA], 2020; Plan Internacional, 2020).

Con relación a los determinantes personales, las y los universitarios han sido obligados a crear nuevos hábitos de estudio, ya que se ha dado una transición de actividades en grupo a individuales y se han establecido nuevas rutinas. Además, es posible que las y los estudiantes tengan inconvenientes para ser autónomos en su aprendizaje debido a problemas de familiarización con las plataformas digitales (Peña, 2020) y a la gestión personal del tiempo. También, puede que la dificultad de comprensión en la modalidad virtual a través de deficiencias en el manejo de software y familiarización con plataformas y bibliotecas virtuales conduzca a una baja calidad de su aprendizaje (Cortéz \& Campos, 2020; Seguimos Virtual, 2020; Torres et al., 2020).

Respecto a los determinantes sociales, varios docentes han reconocido dificultades para usar dispositivos y herramientas virtuales que son necesarias para enseñar, con las cuales no solían interactuar (Hodges et al., 2020). A pesar de las estrategias institucionales para adaptarse a esta realidad, la disminución de la calidad educativa es una preocupación tanto para la educación, en general, (Banco Mundial, 2020) como para la educación superior, en específico (Banco Interamericano de Desarrollo [BID], 2020).

Paralelo a esto, las y los estudiantes han tenido que adaptarse a combinar su carrera universitaria con las circunstancias específicas de su hogar ante la pandemia. No todos los universitarios cuentan con un ambiente libre de ruidos, con una temperatura agradable, buena iluminación y ventilación adecuada, condiciones necesarias para llevar a cabo su proceso de aprendizaje virtual (Raya, 2010). Además, las mujeres se encuentran en particular desventaja en sus hogares y comunidades, ya que en ocasiones sus horarios de estudio son interrumpidos por labores domésticas (Plan Internacional, 2020) y tienen mayor cantidad de horas de cuidado no remunerado y de jornadas laborales que los hombres, lo que se ha agravado con las medidas del confinamiento (CIM-OEA, 2020).

Este proceso de adaptación a la ERE puede repercutir en síntomas emocionales adversos en las y los estudiantes. Antes de la pandemia, estudios ya indicaban que niveles elevados de ansiedad (Palacio \& Martínez, 2007), depresión (Mejía et al., 2011) e insomnio (Suardiaz-Muro et al., 2020) en las y los estudiantes se relacionan negativamente al rendimiento académico. En el caso específico de los síntomas de ansiedad, se reportaba mayor sintomatología durante los exámenes y ante la percepción de sobrecarga de actividades académicas y prácticas (Díaz \& Castro, 2020). Además, existe evidencia de que factores relacionados a los determinantes personales y sociales del aprendizaje, como la metodología implementada por el docente y la relación docente-estudiante, influyen en la salud mental de las y los estudiantes (Cortés, 2017).

\section{Efectos del Covid-19 en la salud mental de las y los estudiantes}

Las pandemias son eventos que generan estrés emocional intenso, lo que lleva a reacciones como el miedo y la ansiedad (Taylor, 2019). La experiencia en epidemias pasadas demuestra que estas pueden desencadenar o exacerbar problemas de salud mental existentes, como trastornos del estado de ánimo y trastornos de ansiedad (Taylor, 2019; Wu et al., 2005). Por ejemplo, en la epidemia por el Síndrome Respiratorio Agudo Grave (SRAS) de 2002 a 2004, síntomas significativos de depresión y ansiedad se identificaron en sobrevivientes (Mak et al., 2009; Wu et al., 2005) 
y personas sometidas a cuarentena (Hawryluck et al., 2004). El público en general se vio menos afectado, pero aquellos cuya familia o amigos habían estado en cuarentena o con sospecha de contagio presentaron mayores síntomas depresivos (Ko et al., 2006).

Hasta el momento, estudios en varios países señalan un impacto negativo en la salud mental de la población bajo el contexto de la pandemia (Hernández, 2020; Marquina \& Jaramillo-Valverde, 2020). Específicamente, se evidencia que en esta pueden desencadenarse síntomas de depresión, ansiedad y estrés (Wang et al., 2020). Un grupo vulnerable a este impacto son las y los universitarios, quienes se ven expuestos a efectos emocionales producto de la incerteza y ansiedad por el futuro, el cambio abrupto del sistema educativo, la inminente recesión económica mundial (Sahu, 2020) y el cambio en las relaciones sociales y actividades de ocio (Son et al., 2020). Investigaciones señalan la presencia de síntomas de ansiedad (Caoa et al., 2020), depresión, estrés (Odriozola-González et al., 2020) y problemas de sueño (Marelli, 2020) significativos en este grupo. Dentro de este, se han identificado como grupos en riesgo de mayores síntomas a las universitarias (Marelli, 2020), quienes tienen un familiar o conocido infectado con Covid-19 (Caoa et al., 2020) y a estudiantes de primer año (Odriozola-González et al., 2020).

En El Salvador, se han reportado síntomas de ansiedad, depresión, estrés (Orellana \& Orellana, 2020) y problemas de sueño (Mena et al., 2020) en la población bajo el contexto de las medidas de restricción de movilidad y la cuarentena. Se sugiere que los grupos especialmente vulnerables son las mujeres, las personas jóvenes (Orellana \& Orellana, 2020), que solamente estudian y están desempleadas (Mena et al., 2020). En el caso de las y los universitarios salvadoreños, existen indicios de la presencia de síntomas somáticos, ansiedad, insomnio, disfunción social y depresión (Castellanos \& Guzmán, 2020), concluyendo que las más afectadas son las mujeres y las personas con edades entre 18 y 23 años (Gutiérrez et al., 2020).

Por tanto, se propone como objetivo general de la investigación establecer las condiciones de educabilidad y el estado de salud mental de los universitarios salvadoreños, bajo la modalidad de actividades académicas virtuales producto de la pandemia por Covid-19. A partir de este, se plantean los siguientes objetivos específicos: (a) describir su percepción de las condiciones de educabilidad; (b) identificar la presencia de síntomas de ansiedad, depresión, estrés e insomnio; (c) relacionar las condiciones de educabilidad con síntomas emocionales, y (d) establecer los perfiles sociodemográficos vulnerables a condiciones de educabilidad y de salud mental adversas.

A continuación, se establece la siguiente pregunta de investigación: ¿Cuáles son las condiciones de educabilidad y el estado de salud mental de los universitarios salvadoreños, bajo la modalidad de actividades académicas virtuales producto de la pandemia por Covid-19?

\section{Metodología}

\section{Tipo de estudio y participantes}

La investigación tiene un diseño cuantitativo transversal de alcance correlacional. Se utilizó un muestreo no probabilístico en cadena o redes de alumnos universitarios de pregrado, mayores de edad y que se encontraban inscritos en el ciclo académico correspondiente al período en el que se recolectaron los datos. Las y los participantes respondieron un cuestionario compartido en línea, procurando un efecto de bola de nieve (Hernández-Sampieri \& Mendoza, 2018).

Los criterios de inclusión de los participantes del estudio fueron: (a) ser mayor de 18 ańos; (b) ser estudiante activo de una universidad o un instituto especializado de El Salvador; y (c) estudiar una carrera de pregrado (técnico, licenciatura, ingeniería o arquitectura). Estos criterios fueron indagados junto al consentimiento informado, por lo que quienes no los cumplieron no participaron en el estudio.

La muestra final está conformada por 1,550 participantes de ambos sexos (38.1\% hombres y $61.9 \%$ mujeres) con una edad promedio de $21.47 \pm 3.74$ años, de por lo menos 25 IES y residentes en los 14 departamentos del país, con predominancia de estudiantes de IES privadas (68.8\%) y de la zona central (76.9\%). Los datos sociodemográficos se describen en la Tabla 1. 
Tabla 1

Datos sociodemográficos de los y las participantes del estudio

\begin{tabular}{|c|c|}
\hline Característica sociodemográfica & Frecuencia $(\%)$ \\
\hline \multicolumn{2}{|l|}{ Sexo } \\
\hline Masculino & $591(38.1)$ \\
\hline Femenino & $959(61.9)$ \\
\hline \multirow{2}{*}{\multicolumn{2}{|c|}{$\begin{array}{l}\text { Edad (media }=21.47 \pm 3.74, \text { mediana }=21, \text { rango }= \\
18 \text { a } 51 \text { ańos })\end{array}$}} \\
\hline & \\
\hline 18 a 19 años & $475(30.5)$ \\
\hline 20 a 21 años & $493(31.6)$ \\
\hline 22 a 23 años & $319(20.5)$ \\
\hline 24 años o más & $272(17.4)$ \\
\hline \multicolumn{2}{|l|}{ Zona geográfica de residencia } \\
\hline Zona central & $1,188(76.9)$ \\
\hline Resto del país & $357(23.1)$ \\
\hline \multicolumn{2}{|l|}{ Tipo de Institución de Educación Superior } \\
\hline Pública & $488(31.2)$ \\
\hline Privada & $1,076(68.8)$ \\
\hline \multicolumn{2}{|l|}{ Año de ingreso a la carrera } \\
\hline 2020 & $478(30.9)$ \\
\hline 2019 a 2018 & $552(33.7)$ \\
\hline 2017 a 2016 & $374(24.1)$ \\
\hline 2015 o menos & $174(11.2)$ \\
\hline \multicolumn{2}{|l|}{ Situación laboral actual } \\
\hline Desempleado o desempleada & $1,075(76.6)$ \\
\hline Estudia y trabaja & $328(23.4)$ \\
\hline \multicolumn{2}{|l|}{ Ingreso en el hogar } \\
\hline Alcanza y pueden ahorrar & $332(22.1)$ \\
\hline Alcanza justo sin dificultades & $810(53.5)$ \\
\hline No alcanza y tienen dificultades & $369(24.4)$ \\
\hline \multicolumn{2}{|c|}{$\begin{array}{l}\text { Personas con las que comparte dispositivo elec- } \\
\text { trónico }\end{array}$} \\
\hline Ninguna persona & $638(43.9)$ \\
\hline Una persona & $402(25.9)$ \\
\hline Dos personas o más & $470(30.2)$ \\
\hline \multicolumn{2}{|c|}{$\begin{array}{l}\text { Ha mejorado el servicio a Internet por las clases } \\
\text { virtuales }\end{array}$} \\
\hline Sí & $961(63.0)$ \\
\hline No & $564(37.0)$ \\
\hline \multicolumn{2}{|l|}{$\begin{array}{l}\text { Ha mejorado su dispositivo electrónico o } \\
\text { adquirido uno nuevo por las clases virtuales }\end{array}$} \\
\hline Sí & $808(53.1)$ \\
\hline No & $714(46.9)$ \\
\hline \multicolumn{2}{|c|}{$\begin{array}{l}\text { Casos activos o pasados de Covid-19 en el grupo } \\
\text { familiar }\end{array}$} \\
\hline Sí & $562(36.3)$ \\
\hline No & $985(63.7)$ \\
\hline
\end{tabular}

\section{Instrumentos}

Datos sociodemográficos. Se preguntó a las y los participantes los datos expuestos en la Tabla 1. La pregunta sobre ingreso en el hogar se retomó del sondeo realizado por Córdova et al., (2017).

Factores generales relacionados a la educabilidad y la salud mental. Esta sección se compuso por tres ítems. Los primeros dos indagaron la percepción de la calidad educativa y del estado de salud mental actuales, en comparación al período previo a la cuarentena, presentadas con opciones de respuestas en una escala tipo Likert de 0 a 5 ( 0 equivale a "mucho peor" y 5 a "mucho mejor", también se incluyó la opción "no sé”). En el tercer ítem se preguntó qué tan probable es cursar el siguiente ciclo si las clases persisten de manera virtual, presentadas con opciones de respuesta en una escala Likert de 1 a 4 (1 equivale a "muy probable" y 4 a "muy improbable", también se incluyó la opción "No sé”).

Escala de condiciones de educabilidad bajo la modalidad de actividades académicas virtuales producto de la pandemia por Covid-19. Esta es una escala tipo Likert de autorreporte ad hoc de 26 ítems que evalúa las condiciones de educabilidad de los universitarios en el contexto de la ERE. Los ítems se presentan con cinco opciones de respuesta, que van desde 0 a 4 (0 significa "muy en desacuerdo" y 4 "completamente de acuerdo"), con una puntuación mínima de 0 y una máxima de 104. La escala se divide en tres subescalas. Algunos ítems han sido retomados y adaptados del estudio \#PulsoEstudiantil (Seguimos Virtual, 2020) con la autorización de los autores.

Los primeros nueve ítems conforman la subescala de "Acceso tecnológico", que indaga el acceso, la facilidad de uso y la calidad de los recursos tecnológicos de las y los estudiantes. Esta tiene una puntuación mínima de 0 y una máxima de 36 . Los siguientes ocho ítems conforman la subescala de "Determinantes personales del rendimiento académico", que informan sobre la percepción de los universitarios sobre su propio aprendizaje, sus hábitos de estudio, su motivación y su administración del tiempo. Tiene 
una puntuación mínima de 0 y una máxima de 32. Los últimos nueve ítems conforman la subescala "Determinantes sociales del rendimiento académico", referidos a la percepción de las y los estudiantes del trabajo de las universidades y de los maestros, así como de las condiciones en el hogar relacionadas a su estudio. Esta subescala tiene una puntuación mínima de 0 y una máxima de 36.

La puntuación máxima de la escala y cada subescala se dividió en tercios, asignándole a cada uno una descripción de las condiciones de educabilidad denominadas: favorables, poco favorables y desfavorables. En la Tabla 2 se muestran las puntuaciones propuestas.

\section{Tabla 2}

Niveles descriptivos de las puntuaciones de la Escala de condiciones de educabilidad y sus subescalas

\begin{tabular}{|c|c|c|c|}
\hline \multirow{2}{*}{ Escala o subescala } & \multicolumn{3}{|c|}{$\begin{array}{c}\text { Puntuaciones de la Escala de condiciones } \\
\text { de educabilidad }\end{array}$} \\
\hline & Desfavorables & $\begin{array}{c}\text { Poco } \\
\text { favorables }\end{array}$ & Favorables \\
\hline Escala total & 0 a 35 & 36 a 68 & 69 a 104 \\
\hline Acceso tecnológico & 0 a 12 & 13 a 24 & 25 a 36 \\
\hline $\begin{array}{l}\text { Determinantes } \\
\text { personales }\end{array}$ & 0 a 9 & 10 a 21 & 22 a 32 \\
\hline $\begin{array}{l}\text { Determinantes } \\
\text { sociales }\end{array}$ & 0 a 12 & 13 a 24 & 25 a 36 \\
\hline
\end{tabular}

La escala mostró una consistencia interna medida por el coeficiente Alpha de Cronbach satisfactoria, con un valor de .876 (ver Taber, 2017). La subescala de Acceso Tecnológico tuvo un alpha de .882 , la de Determinantes personales del rendimiento académico un alpha de .700 y la de Determinantes sociales un alpha de .768 .

DASS-21 (Escalas de Depresión, Ansiedad y Estrés) (Daza et al., 2002). Escala tipo Likert de autorreporte de 21 ítems, que indagan síntomas emocionales de depresión, ansiedad y estrés. La escala tiene cuatro opciones de respuesta, del 0 al 3 (0 significa "nunca" y 3 significa "casi siempre"). En ella se le consigna al participante que describa lo que pasó o sintió en la última semana referente a estas dimensiones, lo que conforma las tres subescalas correspondientes (siete ítems por cada una). Siguiendo las recomendaciones de Roman et al. (2016) sobre su uso como instrumento para tamizaje en universitarios, se utilizó un punto de corte 6 en la subescala de Depresión, 5 en la subescala de Ansiedad y 6 en la subescala de Estrés para identificar a los participantes con síntomas significativos. Para fines descriptivos, los niveles emocionales de las subescalas se describen con los rangos que propone el Black Dog Institute (s.f).

La escala ha mostrado tener adecuadas propiedades psicométricas en la población salvadoreña en general (Mena et al., 2020; Orellana \& Orellana, 2020), en jóvenes salvadoreńos (Gutiérrez-Quintanilla et al., 2020) y en la población universitaria (Antúnez \& Vinet, 2012). En esta aplicación, la escala total mostró una consistencia interna adecuada, con un alpha de .953. La subescala de depresión obtuvo un alpha de .915, la de ansiedad, un alpha de .879; y la de estrés, un alpha de .892 .

Escala Atenas de Insomnio (Soldatos et al., 2000). Escala tipo Likert de autorreporte de 8 ítems basada en los criterios diagnósticos para insomnio no orgánico del CIE-10. La escala tiene cuatro opciones de respuesta, que van desde 0 a 3 (0 significa "ningún problema” y 3 significa "problema muy serio"). Los primeros cuatro ítems de la escala indagan el sueño cuantitativamente, el quinto indaga sobre la calidad global del sueńo y los últimos tres exploran el impacto del insomnio en la calidad del sueño durante el día. Por recomendación de los autores, se utilizó un punto de corte 6 para representar una sintomatología significativa de insomnio no orgánico (Soldatos et al., 2003). Su versión en español ha mostrado tener una adecuada confiabilidad al usarse en la población en general (Monterrosa-Castro, 2013). Se utilizó la validación mexicana de la prueba, adaptada por Nenclares \& Jiménez-Genchi (2005). La escala mostró una consistencia interna adecuada, con un alpha de .863 . 


\section{Procedimiento}

El cuestionario, denominado "Condiciones de educabilidad y salud mental de universitarios salvadoreños bajo la modalidad de actividades académicas virtuales producto de la pandemia por Covid-19”, fue compartido a través de correos electrónicos y mensajes de texto a estudiantes universitarios entre el 11 de noviembre y el 6 de diciembre de 2020, utilizando la plataforma LimeSurvey, alojada en los servidores de la Fundación Pro Educación de El Salvador (FUNPRES). Responder el cuestionario tomó un promedio de 12 minutos.

Como primera pantalla del cuestionario, se presentó un apartado en el que se confirmaban los criterios de inclusión y se solicitaba el consentimiento para que los datos fueran utilizados en la investigación. Si el participante negaba el consentimiento, se daba por finalizado el cuestionario. Luego de completarlo, se animó a los encuestados a compartirlo con personas conocidas que cumplieran los criterios de inclusión. Se recolectaron 1,596 cuestionarios y se descartaron 46 que negaron el consentimiento.

Los datos fueron procesados usando el programa SPSS v.24 (International Business Machines Corporation [IBM], 2016). El tamaño del efecto se reportó a través de la $\mathrm{d}$ de Cohen $(d)$, con la interpretación sugerida por Hernández-Sampieri \& Mendoza (2018) y la interpretación del grado de correlación se realizó atendiendo a los parámetros que expone Akoglu (2018).

\section{Consideraciones éticas}

Se les informó a las y los participantes del propósito de la encuesta y su alcance. Ninguna pregunta era de respuesta obligatoria para finalizar el cuestionario, a excepción del consentimiento. Además, se garantizó el anonimato de las respuestas y se les indicó que podían dejar de contestar el cuestionario cuando lo desearan. Finalmente, la información presentada en este estudio no permite diferenciar a las y los participantes individualmente, sino que los caracteriza utilizando información sociodemográfica.

\section{Resultados}

\section{Percepción de la calidad educativa y la salud mental en comparación al período previo a la cuarentena}

Como se observa en la Tabla 3, la mayoría de los universitarios percibe que la calidad de la educación que recibe y su salud mental es peor o mucho peor en comparación con el período previo a la cuarentena (se excluyó de la pregunta sobre la calidad educativa a quienes comenzaron a estudiar en el año 2020). Además, estas dos percepciones se correlacionaron levemente entre sí $(r=.307, p<.0001)$. Por otro lado, el $85.5 \%$ de las y los estudiantes afirma que es probable que curse el siguiente ciclo de mantenerse las clases de manera virtual, mientras que el $14.5 \%$ lo considera improbable.

\section{Tabla 3}

Percepción de la calidad educativa y salud mental de las $y$ los participantes en comparación al periodo previo a la cuarentena

\begin{tabular}{lccc}
\hline $\begin{array}{l}\text { ¿En comparación al período } \\
\text { previo por la cuarentena, } \\
\text { cómo consideras... }\end{array}$ & $\begin{array}{c}\text { Peor o } \\
\text { mucho } \\
\text { peor }\end{array}$ & Igual & $\begin{array}{c}\text { Mejor o } \\
\text { mucho } \\
\text { mejor }\end{array}$ \\
\hline $\begin{array}{l}\text {..la calidad de la } \\
\text { educación que recibes? }\end{array}$ & $70.8 \%$ & $22.4 \%$ & $6.8 \%$ \\
\hline ...tu salud mental? & $60.0 \%$ & $28.0 \%$ & $12.0 \%$ \\
\hline
\end{tabular}

Nota: la tabla es elaboración propia con base en datos recabados.

La percepción de la calidad de la educación correlacionó débilmente con la edad ( $\mathrm{r}=.186, \mathrm{p}<0.0001)$ y fue moderadamente más favorable en estudiantes de IES privadas que en la universidad pública $[\mathrm{t}(877.274)=-5.645, \mathrm{p}<0.0001, d=.38]$. Esta no difirió significativamente entre hombres y mujeres, año de ingreso a la IES, ni por el ingreso en el hogar. Por otro lado, la percepción del estado de salud mental fue levemente mejor en hombres $[\mathrm{t}(1472)=4.120$, $\mathrm{p}<0.0001, d=.21]$, en quienes trabajan $[\mathrm{t}(1338)=$ $-2.869, \mathrm{p}<0.0001, d=.15]$ y en personas que refieren 
que el ingreso en sus hogares alcanza $[\mathrm{t}(1441)=2.720$, $\mathrm{p}<0.0001, d=.14]$.

\section{Percepción de los universitarios de sus condiciones de educabilidad}

En la Tabla 4 se muestran los resultados de la escala y las subescalas de condiciones de educabilidad de acuerdo con los niveles propuestos. Tanto en la escala total como en las subescalas de Determinantes perso- nales y sociales, la mayoría de las y los participantes se posicionó en niveles poco favorables. Al contrario, la mayoría se ubicó en un nivel favorable en la subescala de Acceso tecnológico. La subescala de Determinantes sociales se correlacionó moderadamente con la de Acceso tecnológico $(r=.469, p<0.0001)$ y Determinantes personales $(r=.580, p<0.0001)$, pero esta última correlacionó levemente con la de Acceso tecnológico $(r=.258, p<0.0001)$.

\section{Tabla 4}

Niveles de las condiciones de educabilidad en los universitarios

\section{Condiciones de educabilidad}

\section{Escala o subescala}

\section{Desfavorables}

Poco favorables

$72.9 \%$

$37.0 \%$

$32.2 \%$

Determinantes personales
$4.6 \%$

$9.0 \%$

$4.6 \%$

Acceso tecnológico
$61.7 \%$
Favorables

$18.1 \%$

$58.4 \%$
$23.3 \%$
$63.2 \%$
Determinantes sociales

$6.0 \%$

Nota: la tabla es elaboración propia con base en datos recabados y los criterios establecidos por los autores.

La comparación entre esta escala y los datos sociodemográficos se reporta en la Tabla 5. Las mujeres, los estudiantes de IES públicas, las personas que han mejorado su conexión y que reportan casos por Covid-19 en su grupo familiar presentaron puntuaciones significativamente menores que sus contrapartes en la escala total y las subescalas, lo que indica peores condiciones de educabilidad. También, la percepción de la calidad educativa comparada con antes de la cuaren- tena correlacionó moderadamente con la escala total $(r=.434, \mathrm{p}<0.0001)$, con la subescala de Determinantes personales $(r=.491, \mathrm{p}<0.0001)$ y la de Determinantes sociales $(r=.470, \mathrm{p}<0.0001)$. La edad solamente correlacionó significativa y negativamente con la escala total $(r=-0.05, p=0.045)$ y con la subescala de Alcance tecnológico $(r=-.202, p<0.0001)$. Al contrario, no hubo diferencias significativas al comparar la situación laboral con esta escala y las subescalas. 
Tabla 5

Estadísticos descriptivos y contrastes bivariados entre la escala de educabilidad y los datos sociodemográficos

\begin{tabular}{|c|c|c|c|c|c|c|c|c|}
\hline \multirow[t]{2}{*}{ Característica } & \multicolumn{2}{|c|}{ Escala total } & \multicolumn{2}{|c|}{ Acceso tecnológico } & \multicolumn{2}{|c|}{$\begin{array}{l}\text { Determinantes perso- } \\
\text { nales }\end{array}$} & \multicolumn{2}{|c|}{$\begin{array}{l}\text { Determinantes } \\
\text { sociales }\end{array}$} \\
\hline & $M(D E)$ & $d$ & $M(D E)$ & $d$ & $M(D E)$ & $d$ & $M(D E)$ & $d$ \\
\hline Promedio general & $55.0(14.9)$ & & $25.4(7.0)$ & & $12.4(5.4)$ & & $14.1(6.4)$ & \\
\hline \multicolumn{9}{|l|}{ Sexo } \\
\hline Masculino & $56.81(15.6)^{*}$ & \multirow{2}{*}{.21} & $26.16(7.2)^{*}$ & \multirow{2}{*}{.17} & $12.8(5.5)^{*}$ & \multirow{2}{*}{.13} & $17.7(6.7)^{*}$ & \multirow{2}{*}{.15} \\
\hline Femenino & $53.9(14.4)$ & & $24.96(6.9)$ & & $12.1(5.3)$ & & $16.8(6.2)$ & \\
\hline \multicolumn{9}{|l|}{ Tipo de IES } \\
\hline Pública & 49.6(13.9) & \multirow{2}{*}{.49} & $24.2(7.2)$ & \multirow{2}{*}{.65} & $11.3(5.0)$ & \multirow{2}{*}{.34} & $14.2(5.9)$ & \multirow{2}{*}{.23} \\
\hline Privada & $57.4(14.7)^{*}$ & & $26.0(6.9)^{*}$ & & $12.6(5.5)^{*}$ & & $18.5(6.2)^{*}$ & \\
\hline \multicolumn{9}{|l|}{ Año de ingreso } \\
\hline 2020 & $55.7(14.2)$ & & $25.6(6.8)$ & & $11.7(5.3)$ & \multirow{2}{*}{.17} & $18.3(5.9)^{*}$ & \multirow{2}{*}{.29} \\
\hline Otro año & $54.7(15.2)$ & & $25.3(7.1)$ & & $12.7(5.4)^{*}$ & & $16.6(6.6)$ & \\
\hline \multicolumn{9}{|l|}{ Ingreso en hogar } \\
\hline Alcanza & $56.67(14.5)^{*}$ & \multirow{2}{*}{.43} & $26.7(6.4)^{*}$ & \multirow{2}{*}{99} & $12.3(5.5)$ & & $17.6(6.4)^{*}$ & \multirow{2}{*}{.28} \\
\hline No alcanza & $49.3(15.0)$ & & $21.2(7.5)$ & & $12.3(5.3)$ & & $15.5(6.4)$ & \\
\hline \multicolumn{9}{|l|}{ Situación laboral } \\
\hline Trabaja & $54.6(14.6)$ & & $24.9(7.2)$ & & $12.8(5.6)$ & & $16.9(6.1)$ & \\
\hline No trabaja & $54.9(15.1)$ & & $25.6(7.1)$ & & $12.2(5.4)$ & & $17.1(6.5)$ & \\
\hline \multicolumn{9}{|l|}{$\begin{array}{l}\text { Ha mejorado su } \\
\text { dispositivo }\end{array}$} \\
\hline $\mathrm{Si}$ & $52.3(14.3)$ & \multirow{2}{*}{.20} & $24.1(6.9)$ & \multirow{2}{*}{.66} & $11.9(5.4)$ & & $16.3(6.2)$ & \multirow{2}{*}{.24} \\
\hline No & $58.0(14.9)^{*}$ & & $27.0(6.7)^{*}$ & & $12.8(5.3)$ & & $18.1(6.5)^{*}$ & \\
\hline \multicolumn{9}{|l|}{$\begin{array}{l}\text { Ha mejorado el } \\
\text { acceso a internet }\end{array}$} \\
\hline $\mathrm{Si}$ & $52.8(14.8)$ & \multirow{2}{*}{.39} & $24.0(7.0)$ & \multirow{2}{*}{.42} & $12.2(5.4)$ & \multirow{2}{*}{.17} & $16.6(6.4)$ & 29 \\
\hline No & $59.0(14.1)^{*}$ & & $28.0(6.1)^{*}$ & & $12.7(5.3)^{*}$ & & $18.1(6.3)^{*}$ & .21 \\
\hline $\begin{array}{l}\text { Casos activos de } \\
\text { COVID-19 }\end{array}$ & & & & & & & & \\
\hline Sí & $52.4(14.4)$ & 26 & $24.5(7.1)$ & 20 & $11.8(5.1)$ & 15 & $16.0(6.2)$ & 26 \\
\hline No & $56.4(15.0)^{*}$ & .20 & $26.0(7.0)^{*}$ & .20 & $12.7(5.5)^{*}$ & 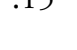 & $17.8(6.4)^{*}$ & .20 \\
\hline
\end{tabular}

Notas: la tabla es elaboración propia con base en datos recabados. Abreviaturas: $M=$ Media aritmética, $D E=$ Desviación estándar, $d=\mathrm{d}$ de Cohen. * Diferencia de medias estadísticamente significativa según prueba t student, $p<.05$. Solamente se reporta el tamańo del efecto de las diferencias estadísticamente significativas. Interpretación del tamańo del efecto expresado en la $d$ de Cohen: .20 o menos, pequeńo; entre .21 y .40, moderado; entre .41 y .60, mediano; entre .61 y .79, considerable; .80 o más, grande (HernándezSampieri \& Mendoza, 2018). 
Descripción de síntomas de depresión, ansiedad, estrés e insomnio

El estado emocional de los universitarios fue mayormente desfavorable. Como lo muestra la Tabla 6, la mayoría manifestó síntomas moderados, severos o extremadamente severos de depresión, ansiedad o estrés. Particularmente, 365 participantes (24.4\%) manifestaron síntomas severos o extremadamente severos de estos tres estados emocionales.

\section{Tabla 6}

Niveles de los estados emocionales de ansiedad, depresión $y$ estrés en la muestra participante

\begin{tabular}{lcccc}
\hline $\begin{array}{c}\text { Estado } \\
\text { emocional }\end{array}$ & Normal & Leve & Moderado & $\begin{array}{c}\text { Severo o } \\
\text { extremadamente } \\
\text { severo }\end{array}$ \\
\hline Depresión & $28.5 \%$ & $10.8 \%$ & $18.6 \%$ & $42.2 \%$ \\
\hline Ansiedad & $39.6 \%$ & $6.2 \%$ & $17.4 \%$ & $36.8 \%$ \\
\hline Estrés & $32.6 \%$ & $10.9 \%$ & $17.8 \%$ & $38.7 \%$ \\
\hline
\end{tabular}

Nota: la tabla es elaboración propia con base en datos recabados y los criterios del Black Dog Institute (s.f).

Utilizando el punto de corte propuesto por Roman et al. (2016) para la escala DASS-21 y el de Soldatos et al. (2003) para la Escala Atenas de Insomnio, en la Tabla 7 se presentan los estudiantes que estarían en riesgo de un estado de ansiedad, depresión, estrés e insomnio clínicamente significativo. En toda la sintomatología explorada, más de la mitad de las y los participantes se encuentra en el grupo en riesgo. $\mathrm{Al}$ analizar los cuatro estados simultáneamente, el $11.5 \%$ no presenta sintomatología clínicamente significativa en ninguno de los estados explorados y el $88.5 \%$ de la muestra está en riesgo por lo menos de padecer uno de ellos. Específicamente, el $45.4 \%$ de las y los participantes presenta sintomatología clínicamente significativa en las cuatro áreas.

\section{Tabla 7}

Niveles clínicamente significativos de sintomatología relacionada a depresión, ansiedad, estrés e insomnio no orgánico en la muestra participante

\begin{tabular}{lcc}
\hline Sintomatología & En riesgo & Fuera de riesgo \\
\hline Depresión & $65.4 \%$ & $34.6 \%$ \\
\hline Ansiedad & $54.1 \%$ & $45.9 \%$ \\
\hline Estrés & $78.8 \%$ & $21.2 \%$ \\
\hline $\begin{array}{l}\text { Insomnio no } \\
\text { orgánico }\end{array}$ & $78.6 \%$ & $21.4 \%$ \\
\hline
\end{tabular}

Nota: la tabla es elaboración propia con base en datos recabados y los criterios de Roman et al. (2020) y Soldatos et al. (2003).

La comparación entre las escalas emocionales y los datos sociodemográficos se reportan en la Tabla 8. Presentaron mayores puntuaciones en las escalas las mujeres, quienes reportan que el ingreso no alcanza, aquellos que han mejorado su dispositivo o el acceso a Internet y quienes reportan casos por Covid-19 en su grupo familiar. Además, la edad de las y los participantes correlacionó negativa y levemente con las subescalas emocionales ( $r$ entre -.056 y $-.151, p<0.0001)$ y la percepción de la salud mental en comparación al período previo a la cuarentena se correlacionó moderada y negativamente con estas escalas ( $r$ entre -.422 y $-.519, p<0.0001)$. Al contrario, las puntuaciones difirieron poco al tomar en cuenta la situación laboral y el estudiar en una IES pública o privada. 
Tabla 8

Estadísticos descriptivos y contrastes bivariados entre las escalas emocionales y los datos sociodemográficos

\begin{tabular}{|c|c|c|c|c|c|c|c|c|}
\hline \multirow{2}{*}{ Característica } & \multicolumn{2}{|c|}{ DASS-21: Depresión } & \multicolumn{2}{|c|}{ DASS-21: Ansiedad } & \multicolumn{2}{|c|}{ DASS-21: Estrés } & \multicolumn{2}{|c|}{ Atenas de Insomnio } \\
\hline & $M(D E)$ & $d$ & $M(D E)$ & $d$ & $M(D E)$ & $d$ & $M(D E)$ & $d$ \\
\hline Promedio general & $9.4(6.4)$ & & $6.6(5.6)$ & & $10.7(5.7)$ & & $10.0(5.3)$ & \\
\hline \multicolumn{9}{|l|}{ Sexo } \\
\hline Masculino & $8.5(6.2)$ & \multirow{2}{*}{.23} & $5.2(5.0)$ & \multirow{2}{*}{.43} & $9.3(5.6)$ & \multirow{2}{*}{.38} & $9.3(10.5)$ & \multirow{2}{*}{.22} \\
\hline Femenino & $10.0(6.4)^{*}$ & & $7.4(5.8)^{*}$ & & $11.5(5.6)^{*}$ & & $10.5(4.9)^{*}$ & \\
\hline \multicolumn{9}{|l|}{ Tipo de IES } \\
\hline Pública & $9.8(6.3)$ & & $6.9(5.5)$ & & $11.2(5.3)^{*}$ & \multirow{2}{*}{.14} & $10.2(4.9)$ & \\
\hline Privada & $9.2(6.4)$ & & $6.4(5.7)$ & & $10.5(5.9)$ & & $9.9(5.4)$ & \\
\hline \multicolumn{9}{|l|}{ Año de ingreso } \\
\hline 2020 & $10.3(6.4)^{*}$ & \multirow{2}{*}{.12} & $7.1(5.8)^{*}$ & \multirow{2}{*}{.14} & $11.2(5.5)^{*}$ & \multirow{2}{*}{.14} & $10.4(5.3)$ & \\
\hline Otro año & $9.1(6.4)$ & & $6.4(5.7)$ & & $10.5(5.9)$ & & $9.9(5.4)$ & \\
\hline \multicolumn{9}{|l|}{ Ingreso en hogar } \\
\hline Alcanza & $9.22(6.4)$ & \multirow{2}{*}{.15} & $6.4(5.6)$ & \multirow{2}{*}{.12} & $10.5(5.8)$ & \multirow{2}{*}{.18} & $9.8(5.3)$ & \multirow{2}{*}{.20} \\
\hline No alcanza & $10.4(6.3)^{*}$ & & $7.2(5.5)^{*}$ & & $11.7(5.5)^{*}$ & & $11.0(5.2)^{*}$ & \\
\hline \multicolumn{9}{|l|}{ Situación laboral } \\
\hline Trabaja & $8.5(6.4)$ & \multirow{2}{*}{.17} & $6.1(5.7)$ & & $10.3(5.8)$ & & $10.0(5.6)$ & \\
\hline No trabaja & $9.7(6.4)^{*}$ & & $6.7(5.6)$ & & $11.0(5.7)$ & & $10.1(5.2)$ & \\
\hline \multicolumn{9}{|l|}{$\begin{array}{l}\text { Ha mejorado su } \\
\text { dispositivo }\end{array}$} \\
\hline $\mathrm{Si}$ & $9.9(6.4)^{*}$ & \multirow{2}{*}{.16} & $7.1(5.8)^{*}$ & \multirow{2}{*}{.21} & $11.2(5.6)^{*}$ & \multirow{2}{*}{.19} & $10.5(5.3)^{*}$ & \multirow{2}{*}{.19} \\
\hline No & $8.9(6.3)$ & & $5.9(5.3)$ & & $10.3(5.8)$ & & $9.5(5.2)$ & \\
\hline \multicolumn{9}{|c|}{$\begin{array}{l}\text { Ha mejorado el acceso a } \\
\text { internet }\end{array}$} \\
\hline $\mathrm{Si}$ & $9.7(6.3)^{*}$ & \multirow{2}{*}{.11} & $7.1(5.8)^{*}$ & \multirow{2}{*}{.31} & $11.0(5.7)^{*}$ & .15 & $10.4(5.3)^{*}$ & 20 \\
\hline No & $8.9(6.4)$ & & $5.5(5.2)$ & & $10.1(5.7)$ & & $9.3(5.1)$ & \\
\hline $\begin{array}{l}\text { Casos activos de } \\
\text { Covid-19 }\end{array}$ & & & & & & & & \\
\hline Sí & $10.1(6.3)^{*}$ & & $7.8(5.8)^{*}$ & & $11.7(5.7)^{*}$ & & $11.0(5.4)^{*}$ & \\
\hline No & $9.0(6.4)$ & .17 & $5.9(5.4)$ & .39 & $10.2(5.6)$ & .24 & $9.5(5.1)$ & .27 \\
\hline
\end{tabular}

Notas: la tabla es elaboración propia con base en datos recabados. Abreviaturas: $M=$ Media aritmética, $D E=$ Desviación estándar, $d=\mathrm{d}$ de Cohen. ${ }^{*}$ Diferencia de medias estadísticamente significativa según prueba t student, $p<.05$. Solamente se reporta el tamańo del efecto de las diferencias estadísticamente significativas. Interpretación del tamaño del efecto expresado en la $d$ de Cohen: .20 o menos, pequeńo; entre .21 y .40, moderado; entre .41 y .60, mediano; entre .61 y .79, considerable; .80 o más, grande (HernándezSampieri \& Mendoza, 2018). 


\section{Relación entre condiciones de educabilidad y síntomas emocionales}

La Escala de condiciones de educabilidad se correlacionó moderada y negativamente con la DASS-21 ( $\mathrm{r}=-.450, \mathrm{p}<0.0001)$ y la Escala Atenas de Insomnio $(\mathrm{r}=-.430, \mathrm{p}<0.0001)$; $\mathrm{y}$ estas dos escalas emocionales se correlacionaron fuertemente entre sí $(\mathrm{r}=.701, \mathrm{p}<0.0001)$.

Además, se aplicó un análisis de regresión múltiple sobre la escala DASS-21 y la Escala Atenas de Insomnio, como se muestra en la Tabla 9. Cada escala fue utilizada como variable dependiente y el resto de las preguntas como variables independientes. Todos los factores fueron sometidos al análisis de regresión y se reportan los estadísticamente significativos. El modelo da la Escala DASS-21 explica el $61.1 \%$ de la varianza (según $\mathrm{R}^{2}$ ajustada), mientras que el modelo de la Escala Atenas de Insomnio explica el 53.4\%.

En los dos modelos, la Escala de condiciones de educabilidad o sus subescalas sirven como factores predictivos significativos. También en ambos la edad es un predictor significativo, aunque en el del DASS-21 la relación es negativa (a más edad menos sintomatología) y en la Escala Atenas de Insomnio es positiva (a más edad mayor sintomatología). Además, las dos escalas fueron predictores significativos entre ellas.

\section{Tabla 9}

Análisis de regresión lineal múltiple de la Escala DASS-21 y la Escala Atenas de Insomnio

\begin{tabular}{|c|c|c|c|c|c|}
\hline & $B$ & ES & $\beta$ & $t$ & $p$ \\
\hline \multicolumn{6}{|l|}{ Escala DASS-21 } \\
\hline Constante & 26.082 & 2.976 & & 8.763 & $<.0001$ \\
\hline Escala Atenas de Insomnio & 1.670 & .081 & .550 & 20.606 & $<.0001$ \\
\hline $\begin{array}{l}\text { Salud mental comparada con } \\
\text { antes de la cuarentena }\end{array}$ & -3.673 & .448 & -.224 & -8.198 & $<.0001$ \\
\hline $\begin{array}{l}\text { Subescala de Determinantes } \\
\text { Personales }\end{array}$ & -.233 & .089 & -.080 & -2.615 & .009 \\
\hline Sexo & 2.770 & .756 & .085 & 3.662 & $<.0001$ \\
\hline Edad & -.296 & .095 & -.073 & -3.101 & .002 \\
\hline $\begin{array}{l}\text { Subescala de Determinantes } \\
\text { Sociales }\end{array}$ & -.179 & .071 & -.072 & -2.515 & .012 \\
\hline \multicolumn{6}{|c|}{$[F(6,744)=195.90, p<.0001] R^{2}$ Ajustado $=0.611$} \\
\hline \multicolumn{6}{|l|}{ Escala Atenas de Insomnio } \\
\hline Constante & 3.462 & 1.087 & & 3.186 & .002 \\
\hline Escala DASS-21 & .223 & .009 & .679 & 24.122 & $<.0001$ \\
\hline $\begin{array}{l}\text { Escala de Condiciones de Educa- } \\
\text { bilidad }\end{array}$ & -.044 & .010 & -.125 & -4.286 & $<.0001$ \\
\hline Edad & .090 & .034 & .067 & 2.646 & .008 \\
\hline IES Pública o privada & .697 & .285 & .064 & 2.448 & .015 \\
\hline \multicolumn{6}{|c|}{$[F(4,744)=213.978, p<.0001] R^{2}$ Ajustado $=0.534$} \\
\hline
\end{tabular}

Notas: la tabla es elaboración propia con base en datos recabados. Se aplicó el método por pasos sucesivos. Las variables independientes de cada modelo han sido ordenadas a partir del peso del coeficiente beta estandarizado. Operacionalización de las variables sociodemográficas categóricas: Salud mental comparada con antes de la cuarentena: 1= Mucho peor, 5= Mucho mejor; IES privadas o pública: 1 = Universidad pública, $2=$ IES privadas; Sexo: $1=$ Hombre, $2=$ Mujer. 


\section{Conclusiones y recomendaciones}

Los resultados indican que la percepción de las y los universitarios salvadoreños encuestados respecto a sus condiciones de educabilidad y su salud mental es poco favorable. De manera general, se evidencia que la mayoría de los universitarios salvadoreños percibe un declive en su salud mental y en la calidad de la educación que reciben. Además, la mayoría presenta condiciones poco favorables de educabilidad y casi la mitad tendría sintomatología significativa de todos los estados emocionales estudiados.

La pandemia ha tenido un efecto notable en las condiciones de educabilidad y la salud mental de los universitarios salvadoreños encuestados, con énfasis en las mujeres, las personas jóvenes, los estudiantes de la IES pública y quienes reportan casos por Covid-19 en su grupo familiar. Esto plantea un llamado de atención a las IES para proveer recursos de apoyo a las y los estudiantes en general, y a estos grupos en específico; además de la necesidad de profundizar más en los factores que están afectando la percepción de las y los estudiantes sobre sus condiciones de educabilidad y realizar los ajustes oportunos. A continuación, se discuten los resultados de acuerdo con los objetivos planteados.

\section{Percepción de los universitarios respecto a sus condiciones de educabilidad}

En primer lugar, siete de cada diez universitarios perciben un declive en la calidad de la educación que reciben durante el ciclo virtual en comparación con antes de la cuarentena. Además, también tienen percepción poco favorable de las condiciones de educabilidad durante la pandemia, a excepción de su capacidad de acceder y usar los recursos tecnológicos, que es en su mayoría satisfactoria. Esto hace eco con las preocupaciones expresadas por organismos internacionales sobre el riesgo de que la calidad de la educación disminuyera durante la pandemia (Banco Mundial, 2020; BID, 2020), que parece coincidir con la percepción de las y los estudiantes. Los resultados sugieren que el impacto de la ERE en la educación superior, expresado a través de las condiciones de educabilidad investigadas, es importante y considerable.

A nivel general, las y los estudiantes perciben poco favorable su capacidad de apropiarse de conocimientos y acceder a los recursos necesarios para el proceso educativo. Bonal \& Tarabini (2016) describen la educación como un medio para disminuir la exclusión social y mejorar la calidad de vida; y a la educabilidad como un foco importante a tomar en cuenta en el proceso, ya que recalca la importante influencia del contexto en el aprendizaje. Es posible que la pandemia no solamente aumente brechas socioeconómicas dadas las condiciones económicas adversas, sino que también tenga un impacto notable en la educación, incluso en quienes se mantengan en el sistema.

Los resultados evidencian que la mayoría adquirió o mejoró sus dispositivos electrónicos $(53.1 \%)$ y su conexión a internet (63.0\%), lo que puede explicar la percepción mayoritariamente favorable del acceso y uso de la tecnología necesaria para la ERE (58.5\%). A más de ocho meses de iniciada la modalidad virtual, parece que las y los estudiantes han adquirido los recursos y las habilidades tecnológicas necesarias para llevar a cabo este proceso. Aun así, existen brechas perceptibles que se vuelven más importantes dado el rol fundamental del acceso a la tecnología en el contexto de la ERE. La relación desfavorable de este acceso y uso tecnológico en mujeres, personas de la universidad pública y (superlativamente) en quienes tienen ingresos insuficientes es preocupante, aunque no novedosa (CIM-OEA, 2020; Plan Internacional, 2020); la brecha digital en El Salvador es un fenómeno muy presente desde antes de la pandemia y está vinculado a poblaciones económicamente desfavorecidas (Lemus \& Villatoro, 2009).

Por otro lado, la puntuación de la subescala de Determinantes personales del rendimiento académico, que evalúa la percepción de las y los estudiantes respecto a su aprendizaje, sus hábitos, su motivación y su administración del tiempo, fue en la que más estudiantes se ubicaron en un nivel poco favorable o desfavorable (93.9\%). Esto parece indicar que las y los estudiantes perciben que las estrategias que han utilizado en esta nueva modalidad de enseñanza no han 
sido exitosas, lo que puede repercutir negativamente en el proceso formativo, incluso cuando las actividades académicas retomen la modalidad presencial. Además, esta subescala fue menos influida que el resto por aspectos económicos y contextuales, como el ingreso en el hogar o la situación laboral, lo que indica un gran componente subjetivo e intrínseco en la percepción del propio aprendizaje.

Dada esta percepción poco favorable de las y los estudiantes con su propio aprendizaje y el rol crucial del acceso a la tecnología en la ERE, resulta notoria la leve correlación entre ambas subescalas. Aunque diversos autores plantean una estrecha relación entre la percepción sobre el aprendizaje y la capacidad de autonomía con la habilidad para usar plataformas digitales (Cortéz \& Campos, 2020; Peńa, 2020), puede que, ante la marcada brecha digital preexistente en el país (Lemus \& Villatoro, 2009), algunos estudiantes hayan tenido que aprender en el contexto pre-pandemia a ser autónomos a pesar del acceso limitado a los recursos tecnológicos requeridos. Esto pudo haberse traducido en un menor impacto de esta disponibilidad tecnológica en su percepción sobre sus capacidades personales, sin demeritar la influencia que este estudio encontró.

Como puente entre estos dos aspectos se encuentran los determinantes sociales, referidos a la percepción de las y los estudiantes del trabajo de las universidades y sus maestros para adaptarse a la modalidad virtual y sobre sus condiciones en el hogar. A pesar de que la percepción de la mayoría se ubicó en un rango poco favorable, esta fue mejor que sobre sus propias capacidades. Los resultados sugieren que muchos estudiantes están insatisfechos con la ejecución de la estrategia virtual por parte de las universidades y además perciben que el ambiente en su hogar no es el adecuado para esta modalidad, ya que se ve interrumpido por ruidos o por trabajo doméstico.

La moderada correlación de las tres subescalas expone la estrecha dependencia entre los factores internos y externos del estudiante, enmarcados en el acceso a la tecnología necesaria en el contexto de la ERE. Aun así, estos tres elementos mantienen suficiente independencia entre sí, lo que alude a la capacidad de la escala y sus componentes de ser interpretada como un todo y por separado. La adecuada confiabilidad de la escala y las subescalas también apoya estos planteamientos.

\section{Síntomas de ansiedad, depresión, estrés e insomnio en estudiantes universitarios}

Por otro lado, seis de cada diez encuestados perciben que su salud mental ha empeorado en comparación con antes de la cuarentena. Además, la elevada correlación entre las escalas de efectos emocionales y el alto poder predictivo mutuo evidencia la elevada comorbilidad de estos padecimientos en los universitarios salvadoreños, relación que puede extenderse también a la población en general (Mena et al., 2020). Los hallazgos también son consistentes con la teoría, que indica que los problemas de sueño son un efecto común dentro del confinamiento (Ramírez-Ortiz et al. 2020) y que estos se relacionan a la aparición de síntomas de ansiedad y depresión (Benetó-Pascual, 2005).

Tomando en cuenta los puntos de corte, nueve de cada diez estudiantes estaría en riesgo de síntomas significativos de alguna de las afectaciones estudiadas y casi la mitad de las cuatro combinadas. Esto coincide con el efecto emocional de epidemias pasadas en la salud mental (Hawryluck et al., 2004; Mak et al., 2009; Wu et al., 2005), con el agravante que la pandemia por Covid-19 parece afectar negativamente a un grupo poblacional no necesariamente expuesto de forma directa al virus.

La salud mental de las y los universitarios en específico parece ser mucho peor que la de la población en general, al compararse con estudios hechos en el país durante la pandemia y que también utilizaron la escala DASS-21 (Mena et al., 2020; Orellana \& Orellana, 2020). Esto puede ser explicado por características propias del ciclo vital en el que se encuentran ellas y ellos.

Por un lado, una menor edad ha sido relacionada a un peor estado de salud mental. El estudio Mental State of the World 2020 (Newson et al., 2021), que analizó datos recolectados antes de la pandemia, expone que las personas de 18 a 24 años tienen un peor estado de 
salud mental que los demás grupos etarios, incluso peor que la de esos grupos cuando eran más jóvenes.

Por otro lado, el final de la adolescencia y el inicio de la adultez temprana es un período en que las amistades son muy importantes, tanto para el proceso de formación de la identidad como para suplir un deseo de pertenencia (Feldman, 2020). Estas relaciones fueron cortadas en el contexto de la pandemia, a pesar de que se encontró un sustituto en espacios virtuales. Además, las y los universitarios ven en su carrera un medio para autodeterminarse a través de un trabajo (Villa, 2016), percepción que pudo haberse catastrofizado en el contexto actual.

Por todo lo dicho, además del impacto adverso de la pandemia común a toda la población, la mayor cantidad de síntomas emocionales en los jóvenes y universitarios se puede explicar por su etapa en el ciclo vital, el impacto del cambio abrupto en la metodología educativa, la incerteza por el futuro (Sahu, 2020) y el corte en las relaciones sociales y las actividades de ocio (Son et al., 2020).

Es importante no patologizar de forma descontextualizada estos resultados. Es posible que las reacciones emocionales adversas sean las esperadas por las medidas de aislamiento y sus consecuencias sociales y económicas (Organización Panamericana de la Salud [OPS], 2020). También, es probable que se trate de problemas de salud mental presentes desde antes de la pandemia (Gutiérrez \& Portillo, 2013), aunque el alto porcentaje de estudiantes que refirieron un peor estado de salud mental en comparación con antes de la cuarentena parece indicar que esta fue un detonante considerable.

\section{Relación entre las condiciones de educabilidad y los síntomas emocionales}

Los resultados también indican que las condiciones de educabilidad y el estado de salud mental están considerablemente relacionados. En primer lugar, la relación entre la percepción de la calidad educativa y de la salud mental en comparación al período anterior a la cuarentena, aunque leve, indica una correspondencia preexistente entre ambas variables
(Cortés, 2017; Mejía et al., 2011; Palacio \& Martínez, 2007; Suardiaz-Muro et al., 2020), que se traslada a las condiciones actuales. En el presente, la moderada correlación entre las escalas de efectos emocionales y la de la Escala de condiciones de educabilidad y el poder predictivo de esta última en los modelos de regresión indica la influencia considerable de las condiciones de educabilidad en la salud mental en el contexto de la pandemia.

En esta relación es importante tomar en cuenta el modelo predictivo de la Escala Atenas de Insomnio. Este explicó más del $50 \%$ de la varianza y aglutinó tanto a la escala DASS-21 como a la Escala de condiciones de educabilidad, virtualmente designándola como un predictor del resto de escalas utilizadas en el estudio. También, los síntomas de insomnio no orgánico (por lo menos cómo son medidos por la escala) pueden ser fácilmente reconocibles por quienes los responden y la escala posee una extensión relativamente corta, lo que facilita su llenado. Por tanto, la exploración de la presencia de insomnio no orgánico (usando esta escala) puede ser un indicador útil de las condiciones de educabilidad y del estado de salud mental de los universitarios salvadoreños.

Además, es notable que, a pesar de las condiciones poco favorables planteadas, la gran mayoría vio probable continuar estudiando el siguiente ciclo (85.5\%). Esto puede responder a la percepción de la educación universitaria como el medio para mejorar los ingresos, el estilo de vida y para propiciar una movilidad social ascendente (Villa, 2016), lo que justificaría el deseo de continuarla a pesar de considerar las condiciones adversas en las que se encuentran.

\section{Grupos vulnerables a peores condiciones de educabilidad y de salud mental}

Las condiciones de educabilidad y los síntomas emocionales fueron consistentemente peores en ciertos grupos. En primer lugar, las mujeres persistentemente presentaron peores condiciones de educabilidad y de salud mental que los hombres en todas las subescalas. Además, presentaron moderadamente mayores dificultades en sus actividades virtuales 
durante la pandemia por cuidar de personas en el hogar o realizar actividades domésticas, lo que alude a la mayor responsabilidad en el hogar que tienen en el contexto de la pandemia (Plan Internacional, 2020; CIM-OEA, 2020). En este contexto, Salamanca \& Rudamas (2020) expresan que las estudiantes universitarias salvadoreñas perciben una inequidad en la distribución de las tareas domésticas y que esto repercute negativamente en su salud mental, lo que se confirma con lo planteado en este estudio.

Por otro lado, los resultados indican que una menor edad se relaciona con un peor estado de salud mental, pero no necesariamente con peores condiciones de educabilidad. Esta relación con el estado de salud mental es incluso predictiva en los modelos de la escala DASS-21, lo que coincide con estudios nacionales en la población general, que también exponen una relación predictiva (Mena et al., 2020; Orellana \& Orellana, 2020). Al respecto, Mena et al. (2020) plantean una triada de vulnerabilidad, asociando un peor estado mental a las personas jóvenes, desempleadas y solteras. Respecto a estas primeras dos características, en esta investigación existió una asociación mediana entre una menor edad y estar desempleado, lo que podría estar relacionado a un peor estado de salud mental, a pesar de que la situación laboral por sí sola no fue un factor muy significativo en el análisis.

Al contrario, las y los estudiantes de la universidad pública presentan un estado emocional relativamente similar al de los de IES privadas, pero peores condiciones de educabilidad que estos, especialmente el acceso a la tecnología. La universidad nacional se ha caracterizado históricamente por una alta demanda de matrícula en comparación con las IES privadas, dado su bajo coste o gratuidad en ciertos casos, pero por un presupuesto insuficiente para cubrir estas demandas (Pintín \& Rivera, 2016). Esta situación, junto a una infraestructura tecnológica deficiente, pueden ser los factores que agravan las condiciones de educabilidad de sus estudiantes.

Otra población vulnerable la componen quienes reportan casos pasados o presentes de Covid-19 en su grupo familiar, lo que indica que el efecto de la enfermedad no solamente atañe a la persona contagiada, sino que también implica emocionalmente a la familia, como ha sucedido en epidemias pasadas (Ko et al., 2006) y en la actual (Caoa et al., 2020). También son notables las peores condiciones de educabilidad en esta población, especialmente en el aspecto contextual. Al respecto, el Programa de las Naciones Unidas para el Desarrollo [PNUD] (2020) ha relacionado la pobreza multimodal, que incluye factores como el hacinamiento, a mayor vulnerabilidad ante el Covid-19, lo podría explicar la relación encontrada.

\section{Limitaciones y futuras líneas de investigación}

Los resultados presentados deben contextualizarse en dos eventos. Por un lado, el período en que se recolectó la información corresponde al de exámenes y evaluaciones finales en las IES salvadoreñas, lo que suele suponer más sintomatología emocional adversa (Díaz \& Castro, 2020). Por otro lado, este período fue muy cercano al paso de dos huracanes en la región centroamericana (Alas, 2020; DW, 2020b), que llevaron a la evacuación de muchas personas y a la suspensión de clases por algunos días. A pesar de que una parte de la sintomatología emocional podría atribuírsele a estos eventos, la percepción muy negativa tanto de la calidad educativa como de la salud mental en comparación con antes de la cuarentena indican que los resultados no dependen necesariamente del contexto de emergencia inmediato, sino que abarcan de forma más amplia la situación de la pandemia y la ERE.

Además, dada la técnica de muestreo no probabilística utilizada, es común que las personas que componen la muestra compartan características y pertenezcan a poblaciones similares (Hernández-Sampieri \& Mendoza, 2018). Aun así, la gran cantidad de participantes y la extendida percepción desfavorable de las condiciones de educabilidad y salud mental parecen indicar que es un fenómeno generalizado a la población universitaria nacional. Por tanto, se recomienda una investigación que compruebe lo planteado utilizando técnicas probabilísticas, enfocándose 
en los grupos vulnerables encontrados y que ahonde en la relación de los estados emocionales descritos, dada su significativa relación y su extendida presencia en la muestra estudiada.

Finalmente, debe considerarse la recolección transversal de los datos al interpretar los modelos predictivos. Se recomienda la ejecución de una investigación longitudinal que retome estos resultados y compruebe su aplicabilidad a lo largo del tiempo.

\section{Referencias}

Akoglu, H. (2018). User's guide to correlation coefficients. Turkish Journal of Emergency Medicine, 18(3), 91-93. https://doi.org/10.1016/j.tjem. 2018.08.001

Alas, L. (2020). Clases virtuales en El Salvador se reanudarán el 9 de noviembre tras lluvias por Eta. El Diario de Hoy. http://bit.ly/3qlU055

Antúnez, Z. \& Vinet, E. (2020). Escalas de depresión, ansiedad y estrés (DASS - 21): Validación de la Versión Abreviada en Estudiantes Universitarios Chilenos. Terapia psicológica, 30(3), 49-55. http://dx.doi.org/10.4067/S0718-480820 12000300005

Banco Interamericano de Desarrollo [BID]. (2020). La educación superior en tiempos de COVID-19: Aportes de la Segunda Reunión del Diálogo virtual con Rectores de Universidades Lideres de América Latina. https://bit.ly/33Zu8DX

Banco Mundial. (2020). Covid-19: impacto en la educación y respuestas de politica pública. https:// bit.ly/2H9pMB1

Barahona, P., (2014). Factores Determinantes del rendimiento académico de los estudiantes de la Universidad de Atacama. Revista Estudios Pedagógicos, 40(1), 25-39. http://dx.doi.org/10.4067/ S0718-07052014000100002.

Benetó-Pascual, A. (2005). Comorbilidad insomnio-depresión. Revista Vigilia-Sueño, 17(2), 116-120. https://www.elsevier.es/es-revista-vigilia-sueno-270-pdf-13085199

Black Dog Institute. (s.f.). DASS 21: Depression Anxiety Stress Scale. https://bit.ly/3ocW9zI
Bonal, X., \& Tarabini, A. (2016). La educabilidad: educación, pobreza y desigualdad. Cuadernos de Pedagogía, No 425. https://bit.ly/3jwX3EA

Caoa, W., Fanga, Z., Houc, G., Hana, M., Xua, X., Donga, J., \& Zhenga, J. (2020). The psychological impact of the COVID-19 epidemic on college students in China. Psychiatry Research, 287. https://doi.org/10.1016/j.psychres.2020. 112934

Castellanos, B., \& Guzmán, J. (2020). Efectos psicológicos que presentan los estudiantes, docentes universitarios y personal administrativo de la Universidad de El Salvador ante la cuarentena domiciliar decretada por la situación de Emergencia del COVID-19 en El Salvador. Universidad de El Salvador.

Comisión Interamericana de Mujeres y Organización de Estados Americanos [CIM-OEA]. (2020). COVID-19 en la vida de las mujeres: Razones para reconocer los impactos diferenciados. https:// bit.ly/2HYdex7

Córdova, R., Rodríguez, M., \& Zechmeister, E. (2017). Cultura politica de la democracia en El Salvador y en las Américas, 2016/17: Un estudio comparado sobre democracia y gobernabilidad. https:// bit.ly/3gEFBxz

Cortés, G. (2017). Factores que intervienen en la reprobación de asignaturas de los estudiantes de primer año de la Facultad de Ingeniería y Arquitectura de la Universidad Centroamericana José Simeón Cañas [Tesis de posgrado, Universidad Rafael Landívar]. https://bit.ly/3gHsJGE

Cortéz, R., \& Campos, M. (2020). Nativos digitales: Tecnologías disponibles y competencias virtuales de los estudiantes UTEC durante la COVID19. Edición Especial, Investigaciones COVID-19. Universidad Tecnológica de El Salvador. http:// hdl.handle.net/11298/1172

Daza, P., Novy, D., \& Stanley, M., A. (2002). The Depression Anxiety Stress Scale-21: Spanish translation and validation with a Hispanic sample. Journal of Psychopathology \& Behavioral Assessment, 24(3), 195-205. https://doi.org/10. 1023/A:1016014818163 
Deutsche Welle [DW]. (2020a). El Salvador decreta cuarentena domiciliar obligatoria por un mes. https://bit.ly/3neOJLX

Deutsche Welle [DW]. (2020b). Tormenta tropical Iota llega a El Salvador y suma una decena de muertos. http://bit.ly/3iaW50T

Díaz, L., \& Castro, J. (2020). Influencia de la ansiedad en el rendimiento académico de estudiantes universitarios [Tesis de grado, Universidad Cooperativa de Colombia]. https://bit.ly/2JMSwS7

Feldman, R. (2020). Desarrollo Psicológico a través de la vida (4 ${ }^{\mathrm{a}}$ ed.). Pearson.

Gutiérrez, J. \& Portillo, C. (2013). La ansiedad y depresión como indicadores de problemas de salud mental en los salvadoreños. Revista Electrónica de Psicología Iztacala, 16(2), 533-557. https://bit.ly/3h9ydZs

Gutiérrez, J., Lobos, M., \& Chacón, E. (2020). Síntomas de ansiedad por la COVID-19, como evidencia de afectación de salud mental en universitarios salvadoreños. Edición especial Investigaciones COVID-19, 44-64. Universidad Tecnológica de El Salvador. https://bit.ly/3i9JDOZ

Gutiérrez-Quintanilla, J., Lobos-Rivera \& Tejada-Rodríguez. (2020). Adaptación psicométrica de las Escalas de Depresión, Ansiedad y Estrés en una muestra de adolescentes salvadoreños. Entorno, (69), 12-22. https://doi.org/10.5377/entorno. v0i69.9556

Hawryluck, L., Gold, W. L., Robinson, S., Pogorski, S., Galea, S., \& Styra, R. (2004). SARS control and psychological effects of quarantine, Toronto, Canada. Emerging infectious diseases, 10(7), 12061212. https://doi.org/10.3201/eid1007.030703

Hernández, J. (2020). Impacto de la COVID-19 sobre la salud mental de las personas. Medicentro Electrónica, 24(3), 578-594. https://bit. ly/323UaVv

Hernández-Sampieri, R. \& Mendoza, C. (2018). Metodología de la investigación: la ruta cuantitativa, cualitativa y mixta. McGraw Hill.

Hodges, Ch., Moore, S., Lockee, B., Trust, T., \& Bond, A. (2020). The Difference Between Emergency Remote Teaching and Online Learning. EDUCAUSE. https://bit.ly/2Sdm77P
Instituto de Acceso a la Información Pública [IAIP]. (2020). Respuestas MINEDUCYT-2020-0214: Matricula Estudiantil de Educación Superior con detalle por Institución, Centro Regional y Sexo 2018. https://bit.ly/3i9zD8w

International Business Machines Corporation [IBM]. (2016). International Business Machines SPSS Statistics para Windows (versión 24.0) [software]. IBM Corporation.

Ko, C., Yen, C., Yen, J., \& Yang, M. (2006). Psychosocial impact among the public of the severe acute respiratory syndrome epidemic in Taiwan. Psychiatry and clinical neurosciences, 60(4), 397-403.https://doi.org/10.1111/j.1440-1819. 2006.01522.x

Lemus, A. \& Villatorio, C. (2009). La brecha digital en El Salvador: causas y manifestaciones [Tesis de pregrado, Universidad Centroamericana José Simeón Cañas]. https://bit.ly/2DzyGXl

Mak, I., Chu, C., Pan, P. Yiu, M., \& Chan, V. (2009). Long-term psychiatric morbidities among SARS survivors. Generay Hospital Psychiatry, 31(4), 318-326. https://doi.org/10.1016/j.genhosppsych.2009.03.001

Marelli, S., Castelnuovo, A., Somma, A., Castronovo, V., Mombelli, S., Bottoni, D., Leitner, C., Fossati, C., \& Ferini-Strambi, L. (2020). Impact of COVID-19 lockdown on sleep quality in university students and administration staff. Journal of Neurology. https://doi.org/10.1007/ s00415-020-10056-6

Marquina, R. \& Jaramillo-Valverde, L. (2020). El COVID-19: Cuarentena y su Impacto Psicológico en la población. Scielo Preprints. https:// preprints.scielo.org/index.php/scielo/preprint/ download/452/560/567

Mediavilla, M. \& Gallego, L. (2016). Condicionantes del rendimiento académico en la escolaridad primaria en Brasil: un análisis multifactorial. Educação \& Sociedade, 37(134), 195-216. https://dx.doi.org/10.1590/ES0101-733020 1683265 
Mejía, C., Gutiérrez, S. \& Perea, E. (2011). Asociación entre depresión y bajo rendimiento académico en estudiantes universitarios. Psicogente, 14(25), 67-75. https://bit.ly/2JVQ0Ja

Mena, F., Molina, C. \& Castillo, Y. (2020). La salud mental de los salvadoreños durante el confinamiento voluntario por el COVID-19. Fundación Pro Educación de El Salvador. https:// funpres.org.sv/la-salud-mental-de-los-salvadorenos-durante-el-confinamiento-voluntariopor-el-covid-19/

Monterrosa-Castro, A., Marrugo-Flórez, M., Romero-Pérez, I., Chedraui, P., Fernández-Alonso, A. M., \& Pérez-López, F. R. (2013). Prevalence of insomnia and related factors in a large mid-aged female Colombian sample. Maturitas, 74(4), 346-351. https://doi.org/10.1016/j.maturitas. 2013.01.009

Nenclares, P. \& Jiménez-Genchi, A. (2005). Estudio de validación de la traducción al español de la Escala Atenas de Insomnio. Salud Mental, 28(5), 34-39. https://bit.ly/2GzWP17

Newson J., Pastukh, V., Sukhoi, O., Taylor, J., \& Thiagarajan, T. (2021). Mental State of the World 2020. Mental Health Million Project, Sapien Labs. https://doi.org/10.5281/zenodo.4603620

Odriozola-González, P., Planchuelo-Gómez, A., Irutia, A., \& Luis-García, R. (2020). Psychological effects of the COVID-19 outbreak and lockdown among students and workers of a Spanish university. Psychiatry Research, 290. https://doi. org/10.1016/j.psychres.2020.113108

Orellana, C. \& Orellana, L. 2020. Predictores de síntomas emocionales durante la cuarentena domiciliar por pandemia de COVID-19 en El Salvador. Actualidades en Psicología, 34 (128), 103-120. https://doi.org/10.15517/ap.v34i1 28.41431

Organización Panamericana de la Salud [OPS]. (2020). Consideraciones psicosociales y de salud mental durante el brote de COVID-19. https://bit.ly/ 2GCspv9
Palacio, J. \& Martínez, Y. (2007). Relación del rendimiento académico con la salud mental en jóvenes universitarios. Psicogente, 10(18), 113-128. https://bit.ly/2W5L5HU

Peña, M. (2020). Análisis sobre la condición actual de los estudiantes de economía en la Universidad de Santo Domingo a raíz de la situación del COVID-19. Universidad Autónoma de Santo Domingo. https://bit.ly/2HOYDnt

Pérez, C. (2020). El gran test de las clases 'online’. La Tercera. https://bit.ly/3itUlOT

Pintín, A. \& Rivera, T. (2016). Evaluación de la inversión en educación superior universitaria en el marco del modelo neoliberal (1999-2012) [Tesis de grado, Universidad de El Salvador]._ https:// bit.ly/3jo3fQ4

Plan Internacional. (2020). Informe final de investigación "Sondeo sobre múltiple jornada y riesgos de violencia sexual y de género en mujeres, adolescentes y niñas durante cuarentena por COVID-19". https://bit.ly/3nrTJMS

Presidencia de El Salvador. (2021). Gobierno suspende inicio de clases presenciales ante la tendencia de incremento de casos de COVID-19 en las últimas semanas. http://bit.ly/3khxrgd

Programa de las Naciones Unidas para el Desarrollo [PNUD]. (2020). Covid-19 y vulnerabilidad: una mirada desde la pobreza multidimensional. https://bit.ly/3aHQz2H

Ramírez-Ortiz, J., Fontecha-Hernández, J. \& Escobar-Córdoba, F. (2020). Efectos del aislamiento social en el sueño durante la pandemia COVID-19. Scielo preprints. https://doi.org/ 10.1590/SciELOPreprints.801

Raya, E. (2010). Factores que intervienen en el aprendizaje. Temas para la educación, 7. https://bit.ly/ 39RGbpK

Rodelo, M. \& Castro, S. (2016). Reflexiones sobre la educabilidad: Elemento fundamental en el proceso educacional del desarrollo humano. Cultura Educación y Sociedad, 7(2), 77-93. http://bit.ly/3cQakaT 
Roman, F., Santibáñez, P. \& Vinet, E. (2020). Uso de las Escalas de Depresión Ansiedad Estrés (DASS-21) como Instrumento de Tamizaje en Jóvenes con Problemas Clínicos. Acta de Investigación Psicológica, 6(1), 2325 - 2336. https:// doi.org/10.1016/S2007-4719(16)30053-9

Sahu, P. (2020). Closure of Universities Due to Coronavirus Disease 2019 (COVID-19): Impact on Education and Mental Health of Students and Academic Staff. Cureus, 12(4), e7541. https:// dx.doi.org/10.7759\%2Fcureus.7541

Salamanca, D., \& Rudamas, M. (2020). Invisibles, limitadas y no remuneradas: mujeres universitarias y trabajo de cuidados en pandemia. Alharaca. http://bit.ly/3cLfepD

Seguimos Virtual. (2020). \#PulsoEstudiantil: estudio revela el impacto del COVID-19 en la vida de las y los estudiantes chilenos. https://bit.ly/3j0I192

Soldatos, C., Dikeos, D. \& Paparrigopoulos, T. (2003). The diagnostic validity of the Athens Insomnia Scale. Journal of Psychosomatic Research, 55(3), 263-267. https://doi.org/10.1016/s0022-3999 (02)00604-9

Soldatos, C., Dikeos, D., \& Paparrigopoulos, T. (2000). Athens Insomnia Scale: validation of an instrument based on ICD-10 criteria. Journal of Psychosomatic Research, 48(6), 555-560. https://doi.org/10.1016/s0022-3999 (00)00095-7

Son, C., Hegde, S., Smith, A., Wang, X., \& Sasangohar, F. (2020). Effects of COVID-19 on College Students' Mental Health in the United States: Interview Survey Study. Journal of Medical Internet Research, 22(9), e21279. https:// doi. org/10.2196/21279

Suardiaz-Muro, M., Morante-Ruiz, M., Ortega-Moreno, M., Ruiz, M., Martín-Plasencia, \& P., Vela-Bueno. A. (2020). Sueño y rendimiento académico en estudiantes universitarios: revisión sistemática. Revista de Neurología, 71(02), 43-53. https://doi.org/10.33588/rn.7102. 2020015
Taber, K. (2017). The use of Cronbach's alpha when developing and reporting research instruments in science education. Research in Science Education, 48, 1273-1296. https://doi.org/10.1007/ s11165-016-9602-2

Taylor, S. (2019). The Psychology of Pandemics: Preparing for the Next Global Outbreak of Infectious Disease. Cambridge Scholars Publishing.

Tipismana, O. (2019). Factores de Resiliencia y Afrontamiento como Predictores del Rendimiento Académico de los Estudiantes en Universidades Privadas. REICE. Revista Iberoamericana Sobre Calidad, Eficacia Y Cambio En Educación, 17(2). https://doi.org/10.15366/reice2019.17.2.008

Tobar, L. (2020). Los retos de la educación en El Salvador ante las pandemias liquidas. Diario Digital Contrapunto. https://bit.ly/34Xw7b9

Torres A., Rivera I., Molina R., Marcano J., Rodríguez, L. Rivera M., Cruz, V. \& Nina, D. (2020). Brecha digital, aprendizaje y salud mental: Experiencias y retos del estudiantado de la Universidad de Puerto Rico en Humacao (UPRH) ante el COVID-19. Universidad de Puerto Rico en Humacao, Instituto Transdisciplinario de Investigación-Acción Social (ITIAS). https://bit.ly/ $2 \mathrm{HRbXrD}$

Villa, L. (2016). Educación superior, movilidad social y desigualdades interdependientes. Universidades, 88, 51-62. https://bit.ly/3tFjcq3

Wang, C., Pan, R., Wan, X., Tan, Y., Xu, L., Ho, C., \& Ho, R. (2020). Immediate psychological responses and associated factors during the initial stage of the 2019 coronavirus disease (COVID19) epidemic among the general population in China. International journal of environmental research and public health, 17(5), 1729. https:// doi.org/10.3390/ijerph17051729

Wu, K., Chan, S., \& Ma, T. (2005). Posttraumatic Stress, Anxiety, and Depression in Survivors of Severe Acute Respiratory Syndrome (SARS). Journal of Traumatic Stress, 18(1), 39-42. https:// doi.org/10.1002/jts.20004 